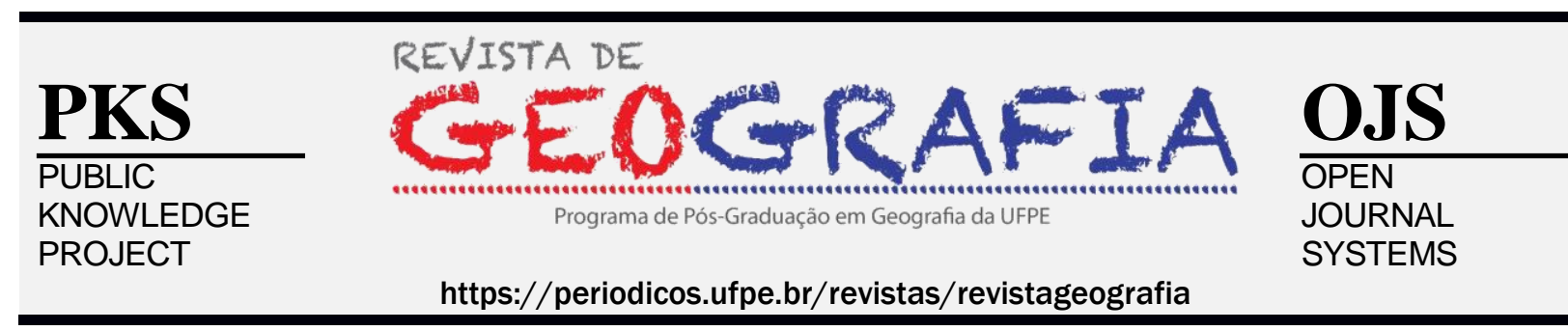

\title{
BACKGROUND GEOQUÍMICO DE SEDIMENTOS DE FUNDO DE ESTUÁRIOS COMO SUBSÍDIO AO GERENCIAMENTO AMBIENTAL: UMA REVISÃO
}

\author{
Natália Micheli Mendes ${ }^{1}$, João Adauto de Souza Neto², Germano Melo Jínior ${ }^{3}$ \\ ${ }^{1}$ Professora do colegiado de Geografia da UNIVASF atuando nas área de Geografia Física (Geomorfologia e \\ Geologia).E-mail: natalia.micheli@univasf.edu.br \\ ${ }^{2}$ UFPE - Professor do Departamento de Geologia.Email: adautou@ufpe.br \\ ${ }^{3}$ UFRN - Professor do departamento de Geologia.Email: germano@ geologia.ufrn.br
}

Artigo recebido em 21/12/2018 e aceito em 14/06/2019

\begin{abstract}
RESUMO
$\mathrm{O}$ crescimento industrial e populacional tem influenciado nas concentrações de elementos maiores, menores e traços nos sedimentos estuarinos e impactado esses ecossistemas. A determinação do background geoquímico é fundamental para subsidiar o monitoramento desses contaminantes, tendo como base as características físicas (e.g. substrato rochoso, clima) regionais. Diversos estudos vêm buscando estabelecer esses índices para identificar áreas com possíveis indícios de contaminação. O presente trabalho apresenta aspectos da importância dos ecossistemas estuarinos tropicais e o papel dos sedimentos de fundo destes como suportes geoquímicos para os elementos contaminantes, enfatizando a necessidade de se estabelecer o background geoquímico como subsídio às ações de gerenciamento desses ambientes.
\end{abstract}

Palavras-chave: Geoquímica ambiental; estuários; Gestão.

\section{GEOCHEMICAL BACKGROUND IN TROPICAL ESTUARINE SEDIMENTS:SUBSIDY TO ENVIRONMENTAL MANAGEMENT}

\begin{abstract}
Industrial and populational growth has influenced the concentration of major, minor and trace elements in estuarine sediments and affected these ecosystems. Determination of geochemical background is paramount for supporting monitoring these contaminants, taking into consideration regional physical characteristics (e.g. rock substrate, climate). Several studies have sought establishing these indices aimed at identifying possible indication of contamination. This paper presents aspects regarding the importance of tropical estuarine ecosystems and the role of their bottom sediments as geochemical sink of contaminant elements, with emphasis on the need for the establishing of geochemical background as a tool for management efforts of these environments.
\end{abstract}

Keywords: Environmental Geochemistry, estuary, Management. 


\section{INTRODUÇÃo}

As atividades humanas interferem diretamente sobre os ciclos geoquímicos de elementos maiores, menores e traços presentes no ambiente, o que pode ocasionar sua contaminação. $\mathrm{O}$ descarte inadequado de substâncias contaminantes, a partir de fontes industriais, tem contribuído para a alteração das condições naturais de diversos estuários no Brasil e no mundo (LI et al., 2012; SANDERS et al., 2011).

A depender dos aspectos hidrodinâmicos e das condições ambientais estuarinas esses elementos tendem a acumular-se nos sedimentos na parte inferior da coluna de água. No caso de níveis tóxicos serem atingidos os organismos bentônicos podem ser afetados bem como a cadeia alimentar, aumentando com isso a possibilidade de ameaça à saúde humana da população local (LOURIÑOCABALA et al., 2011).

Diversos contaminantes inorgânicos, como por exemplo, os metais, têm recebido atenção devido ao risco ambiental que representam. Contudo, nem sempre as altas concentrações desses metais, em sedimentos estuarinos, implicam na ocorrência de contaminação. Isso pode apenas refletir as características geológicas locais (MIRLEAN et al., 2011).

No litoral do estado de Pernambuco, por exemplo, em um estudo realizado por Mendes (2015), foram encontradas concentrações de arsênio (As) elevadas apenas nos estuários do litoral sul, diferente do que foi observado no litoral norte. Tal fato foi relacionado à fonte geogênica - rochas vulcânicas.

Cabe destacar que os setores sul e norte do litoral pernambucano estão dispostos em bacias sedimentares distintas, bacia Pernambuco e bacia Paraíba, respectivamente. Na primeira ocorreram eventos de vulcanismo (NASCIMENTO, 2003), sendo que tais manifestações não ultrapassaram o Lineamento Pernambuco (LIMA FILHO, 1998), ou seja, não ocorreu na bacia Paraíba.

Com isso, estabelecer o background geoquímico para distinguir as concentrações naturais das antropogênicas de elementos químicos diversos em sedimentos estuarinos vem se impondo como cada vez mais necessário para o desenvolvimento de planos de políticas ambientais e de saúde pública. De modo que as estratégias de gerenciamento não sub ou superestime o grau de contaminação dos ambientes estuarinos trazendo prejuízos ambientais e/ou econômicos.

Neste artigo são apresentados aspectos quanto à importância dos ecossistemas estuarinos e o papel dos sedimentos de fundo destes como suportes geoquímicos para elementos contaminantes, 
enfatizando a necessidade de se estabelecer o background geoquímico como subsídio à gestão desses ambientes.

\section{IMPORTÂNCIA DOS ESTUÁRIOS}

Os estuários são ambientes de grande importância ecológica, econômica e social. Servem como áreas de alimentação e refúgio para peixes marinhos juvenis que se reproduzem no mar, compreendem áreas de alimentação utilizadas sazonalmente por adultos de peixes marinhos e como habitat definitivo para outras espécies (AMORIM et al., 2017; ABLE e FAHAY, 2010; POTTER et al., 2015).

A situação de interface entre zonas terrestres e aquáticas e entre os ambientes fluviais e marinhos fazem dos estuários um meio muito específico cujos serviços ecossistêmicos oferecidos são de valor inestimável. Dentre esses serviços pode-se citar: a filtração da água e a proteção do habitat.

Os habitats associados aos estuários (e.g. restingas e manguezais) agem como grandes filtros biogeoquímicos reciclando nutrientes e outras substâncias que chegam até ele em concentrações elevadas, antes de transferi-las ou exporta-las para a zona marinha (CHESTER e JICKELLS, 2012). Os estuários e suas áreas úmidas circundantes também funcionam como zonas de amortecimento (VAIPHASA et al., 2006).

Eles protegem as áreas costeiras, habitats terrestres e as comunidades humanas de inundações e tempestades. Quando ocorrem inundações, os estuários muitas vezes agem como grandes esponjas, absorvendo o excesso de água. Além disso, também protegem córregos, canais de rios e praias costeiras da erosão causada pelos ventos e água.

Ainda há que se destacar sua elevada produtividade biológica, elevado número de habitats e grande diversidade de peixes e avifauna que os utiliza como local de nidificação, alimentação ou abrigo. Uma das razões que justificam a alta produtividade dos ecossistemas estuarinos é o fato das águas do sistema carregarem nutrientes da bacia circundante (DAY et al., 2013).

Por outro lado, além de nutrientes, essa mesma água, muitas vezes traz consigo todos os poluentes que foram introduzidos nas terras da bacia hidrográfica. Por este motivo, os estuários formam alguns dos ecossistemas mais férteis da Terra, mas também podem ser alguns dos mais poluídos.

Além do valor ambiental, os estuários possuem igualmente importante valor econômico, paisagístico e social fornecendo diversos bens e serviços. Diversas são as atividades antrópicas desenvolvidas nessas áreas. Dentre elas estão: a pesca tradicional, a coleta de bivalves, a 
carcinicultura, a agricultura, as atividades portuárias e industriais, a pesca comercial e recreativa, a exploração de sedimentos, a exploração e produção energética, atividades de turismo, recreio, lazer e desporto, dentre outras.

Somado a essas atividades está a ocupação antrópica sob suas diversas formas, a saber: urbana, industrial, portuária, turística, agrícola etc. Com isso, impactos diretos e indiretos ocorrem ao longo dos estuários, seu leito, margens e ecossistemas associados.

Os estuários agem como filtros biogeoquímicos reciclando nutrientes e outras substâncias que chegam até eles em concentrações elevadas, antes de transferi-las ou exportá-las para a zona marinha (CHESTER e JICKELLS, 2012). O conceito de filtro estuarino é baseado no fato de que a mistura de água doce e salgada resultará no estabelecimento de fortes gradientes físico-químicos em um ambiente submetido a variações contínuas no fornecimento de matéria e energia.

Tais gradientes são as forças condutoras desse filtro. O filtro estuarino atua sobre o material particulado e dissolvido que são transportados pelo rio, podendo haver modificação e retenção dentro da zona de mistura das águas (CHESTER e JICKELLS, 2012). Essas modificações ocorrem via processos físicos, químicos e biológicos envolvendo mudanças na especiação dos componentes encontrados na água.

Desse modo, o material particulado pode agir tanto como uma fonte de componentes dissolvidos, que são liberados dentro da solução, como um aprisionador de componentes dissolvidos, que são removidos da solução. Uma vez que toda água existente em um estuário flui para fora do mesmo em uma escala de dias ou semanas, apenas o sedimento agirá como um retentor dos elementos que são trazidos pelo rio. Entretanto, os sedimentos não são um reservatório estático dentro do sistema estuarino.

Diferente disso eles são submetidos a uma infinidade de processos físicos, químicos e biológicos, podendo resultar na reciclagem dos componentes depositados de volta a água. Estes processos de reciclagem incluem (CHESTER e JICKELLS, 2012): a difusão química de componentes a partir da água intersticial; o deslocamento da água intersticial para a coluna d’água, e; a ressuspensão dos sedimentos de superfície devido às marés, podendo levar à sua transferência de um local para outro. A mobilização de elementos contaminantes presentes nos sedimentos estuarinos é potencialmente perigosa para a manutenção do ecossistema. Contaminantes como os metais, por exemplo, em grande concentração, merecem atenção, uma vez que permanecem por longos períodos no ambiente, principalmente nos sedimentos. 


\section{ELEMENTOS QUÍMICOS EM AMBIENTE SEDIMENTAR ESTUARINO E SUPORTES GEOQUÍMICOS}

De forma geral os processos que se desenvolvem ao longo das bacias hidrográficas tendem a convergir para os estuários. A presença de atividades humanas leva a esses ecossistemas elevadas concentrações de materiais antrópicos que podem ocasionar danos ambientais (OLIVEIRA e MARINS, 2011). Os sedimentos estuarinos possuem fontes distintas que vão desde a lixiviação da bacia de drenagem, plataforma continental, atmosfera, bem como a erosão dentro do próprio corpo estuarino e da atividade biológica (MIRANDA et al., 2002).

Os sedimentos, em particular, desempenham um papel importante no diagnóstico da condição ambiental de um sistema estuarino. Tal importância se deve a sua grande capacidade de acumular contaminantes bem como de remobilizá-los (ALEXAKIS, 2008) o que lhes confere um caráter mais permanente dos efeitos provenientes da contaminação.

Diversas são as funções dos sedimentos no meio aquático, como: efeito memória em ambientes de deposição, uma vez que as camadas de deposição são temporal e sequencialmente acumuladas; suporte à vida, pois além de representar uma parte essencial do ecossistema aquático formando uma variedade de habitats e ambientes, fornece nutrientes para os organismos aquáticos; fonte secundária, com mobilização de partículas contaminadas e subsequente liberação de contaminantes com ressuspensão natural ou artificial dos sedimentos e; reservatório final de contaminantes, por meio da capacidade de imobilizar elementos potencialmente perigosos, como por exemplo, os metais pesados (FÖRSTNER, 2004).

Nos sedimentos os elementos químicos distribuem-se de forma heterogênea nos diferentes tamanhos de seus grãos e, de forma geral, uma grande diferença na concentração total de metais é observada para uma mesma amostra (JESUS et al., 2004). Normalmente a fração onde há maior concentração de metais é a inferior a $63 \mu \mathrm{m}$.

A reatividade das partículas de sedimentos estuarinos pode mudar ao longo de intervalos espaciais curtos devido às rápidas mudanças na salinidade, $\mathrm{pH}$, e condições redox (TURNER e MILLWARD, 2002). Com isso a precipitação dos elementos contaminantes (e.g. As, Pb, Cd) é influenciada por esses parâmetros assim como pela presença de óxi-hidróxidos de Fe e Mn, matéria orgânica e argilominerais (KENNISH, 1995; LACERDA e MARINS, 1997). 
As perturbações que ocorrem nos sedimentos podem conduzir a alterações nas propriedades químicas desses, estimulando a mobilização de contaminantes. Dentre os fatores que governam a mobilidade dos elementos em solução no ambiente estuarino Calmano e Forstner (1993) indicam o conjunto $\mathrm{pH}$ e Eh como o possivelmente mais importante, uma vez que mudanças nesses parâmetros podem acelerar dessorção, particionamento, degradação bacteriana e a oxidação de contaminantes.

Como exemplo pode se citar a mobilidade do arsênio. Esta ocorre em ambientes oxidantes, sendo muito baixa em ambientes redutores (REIMANN e CARITAT, 1998). Já a mobilidade do Cd é média em ambientes oxidantes, sendo muito baixa em ambientes redutores, além disso, o Cd é muito solúvel a pH baixo (REIMANN e CARITAT, 1998).

Quanto à salinidade, analisando-se a variação da concentração da espécie contaminante de interesse em função desta, é possível, compreender os processos químicos relacionados à propriedade conservativa de tais espécies em regiões de mistura estuarina (CHURCTH e SCUDLARK, 1998).

No que concerne à matéria orgânica, a sua decomposição, associada aos sedimentos, provoca modificações que influenciam no ciclo biogeoquímico de vários elementos, determinando a forma na qual os mesmos encontram-se ali presentes. Essas modificações consistem de reações diagenéticas capazes de produzir decomposição de matéria orgânica reduzida, material húmico e quelação de metais (TAN, 2014).

Entretanto, tem sido observado em sedimentos muito salinos que a matéria orgânica possui uma importância secundária na retenção/complexação de metais comparada àquela exercida pelos óxidos de Mn/Fe e aos sulfetos (PERIN et al., 1997).

Quanto aos óxidos de Fe e Mn, estes têm influência direta na mobilidade dos elementos. Estes se dissolvem a medida que o Eh decresce e reprecipitam à medida que o Eh aumenta (JENNE, 1968). O mesmo autor afirma ainda que em solos, sedimentos e água, os óxidos são os principais controladores de metais pesados. Assim metais como $\mathrm{Cu}, \mathrm{Co}, \mathrm{Zn}$ e Ni, solúveis em condições levemente ácidas, podem ser agregados nos precipitados de $\mathrm{Fe}, \mathrm{Al}$ e $\mathrm{Mn}$, deixando de estar solubilizados na água e passando a incorporar os sedimentos de drenagem.

Os hidróxidos de Fe, bem como materiais ricos em carbonatos podem, por exemplo, controlar a mobilidade do As, causando o acúmulo através de processos de sorção ou co-precipitação (ROMERO et al., 2004). Cabe salientar que $\mathrm{Fe}\left(2^{+}\right.$e $3^{+}$pode formar hematita $\left(\mathrm{Fe}_{2} \mathrm{O}_{3}\right)$ e magnetita 
$\left(\mathrm{Fe}_{3} \mathrm{O}_{4}\right)$ em condições mais oxidantes ou mais redutoras. Assim, a relação Eh versus solubilidade de metais é complexa.

Os argilominerais desempenham um papel fundamental nos processos estuarinos. Isso devido a uma propriedade importante das argilas, a atividade química de sua superfície, que surge devido à carga superficial. $\mathrm{O}$ fato dessas partículas (e.g. ilita, caulinita) possuírem a capacidade de trocarem seus cátions com uma solução, interfere diretamente na mobilidade dos metais em ambientes estuarinos (BIANCHI, 2007).

Esta influência ocorre em função de tais elementos ficarem retidos e fixados nos argilominerais, que, por conseguinte constituem um reservatório importante para os contaminantes. Havendo perturbação hidrodinâmica, por exemplo, as argilas podem ser remobilizadas deixando- os livres.

\section{BACKGROUND GEOQUÍMICO}

As atividades antrópicas são responsáveis pela introdução de diversos contaminantes (p.ex. As, $\mathrm{Pb}$, $\mathrm{Cd}, \mathrm{Hg}$ ) nos compartimentos ambientais. Esse acréscimo de cargas contaminantes no ambiente traz consigo a necessidade de se entender que consequências tais interferências podem acarretar ao ambiente. Com isso as investigações relacionadas ao processo de contaminação do ar, água, solo e sedimentos têm sido crescentes tornando o uso do termo background geoquímico cada vez mais importante nos estudos ambientais (MATSCHULLAT et al., 2000).

Nas ciências ambientais o background geoquímico é considerado como a medida relativa para distinção entre as concentrações naturais de um elemento ou composto e as concentrações influenciadas por ações antrópicas (MATSCHULLAT et al., 2000). Outra definição é sugerida por Galuszka (2006), onde o mesmo é conceituado como a concentração teoricamente natural de um determinado elemento observando a variável temporal e espacial.

Alguns métodos são utilizados para a determinação do background geoquímico. Gałuszka e Migaszewski (2011) os classificam em métodos: direto (geoquímico), indireto (estatístico) e integrado. O método direto se utiliza de valores médios obtidos através de amostras representativas do período pré-industrial, onde se observa o aspecto histórico (LUIZ-SILVA et al., 2006; BIRCH, 2013), ou obtidos em áreas preservadas, onde se observa o aspecto contemporâneo (DEPAULA e MOZETO 2001; SABADINI- SANTOS et al., 2009). 
Como vantagens do uso do método direto pode ser citado o fato de o background geoquímico ser estabelecido de forma relativamente simples, sendo para isso utilizado as médias ou medianas das concentrações dos elementos. Além disso, não há necessidade de processamento dos resultados originais.

Por outro lado, tal método exige um profundo conhecimento sobre o comportamento geoquímico dos elementos estudados e sobre as condições ambientais dominantes, incluindo o entendimento das condições paleoambientais e sedimentológicas, que nem sempre são facilmente entendidas (GALUSKA, 2007).

Há ainda o fato de que a coleta de amostras pré-industriais e materiais datados, para algumas áreas, não garante que eles não passaram por impactos antropogênicos. A exemplo disso há os estudos de Hong et al. (1994) e Hong et al. (1996), onde foram encontrados níveis elevados de Pb que remonta para os antigos períodos gregos e romanos (encontrados em núcleos de gelo da Groenlândia), bem como de $\mathrm{Cu}$ desde os tempos romanos e medievais.

No que concerne aos dados obtidos em áreas preservadas, a utilização destes é frequentemente criticada. Isso porque de forma geral há subjetividade nos critérios de seleção e na decisão das áreas a serem estudadas quanto a seu nível de preservação (GALUSKA, 2007). Somado a isso os custos são bastante elevados e há uma carga pesada de trabalho em laboratório.

O método indireto (estatístico) baseia-se em técnicas estatísticas (computacionais e gráficas), que visam eliminar os valores extremos de distribuição da população estatística (considerados como antropicamente influenciados) (MATSCHULLAT et al., 2000), sendo o background representado por concentrações não anômalas.

Tal método é criticado pelos geoquímicos por negligenciar a importância dos processos naturais que influenciam a distribuição de elementos ou de compostos químicos no ambiente e por não considerar a incerteza de fases de tratamento da amostra, incluindo a amostragem, a preparação da amostra e análise química (PASLAWSKI e MIGASZEWSKI, 2006).

Vários métodos estatísticos são usados para avaliar o background geoquímico (MATSCHULLAT et al., 2000; REIMAM e GARRET, 2005). A revisão detalhada de tais métodos foi publicado por Reimann et al. (2005). Seu ponto de vista é que a construção de boxplots e diagramas de frequência cumulativa, juntamente com informações obtidas a partir de mapas geoquímicos, são as melhores maneiras de se determinar o background geoquímico. 
O método integrado é uma combinação do método direto e indireto, no qual as amostras são coletadas em áreas preservadas e os resultados são submetidos a cálculos estatísticos (GALUSZKA, 2006). As principais vantagens desse método estão em: levar em consideração o conhecimento especializado sobre o comportamento dos elementos no meio ambiente; utilizar uma abordagem estatística, influenciando a maior precisão da avaliação do background; permite avaliar o background em escalas regionais e locais.

Como desvantagens podem ser apontadas a subjetividade da seleção da área de estudo; os custos elevados e a carga de trabalho de laboratório pesado; além da necessidade de conhecimentos especializados.

Independente do método escolhido para se estabelecer o background geoquímico é importante perceber que é impossível determiná-lo com uma certeza absoluta. Uma vez que este representa uma gama teórica de valores que podem ser obtidos com diferentes métodos (GALUSKA, 2006). Assim, é de suma importância que para se obter valores passíveis de comparação, um método geoquímico específico e confiável seja usado de forma que seu uso no âmbito do gerenciamento ambiental seja efetivo.

A legislação nacional vem buscando estabelecer tais índices, apesar de até o momento haver apenas valores para sedimentos a serem dragados, na Resolução do CONAMA N 454/2012. Tal resolução estabelece valores orientadores para alguns contaminantes no material a ser dragado, com base na experiência internacional, utilizando valores estabelecidos de outros países.

Para efeito de classificação do material a ser dragado, são definidos critérios de qualidade que se baseiam em níveis:

Nível 1: Valor limiar abaixo do qual se prevê baixa probabilidade de efeitos adversos à biota;

Nível 2: valor limiar acima do qual se prevê efeito adverso à biota.

Para determinação da concentração de metais em sedimentos, o CONAMA (2012) indica que a metodologia analítica para extração dos metais das amostras consista em ataque com ácidos minerais e aquecimento, conforme EPA SW 846: método 3050B - exceto Hg (U.S. EPA, 1996) e 3051A - todos e metais e semi-metais (U.S. EPA, 2007), 7471B - Hg (U.S. EPA, 2007b) ou metodologia similar.

Os valores orientadores adotados baseiam-se na experiência internacional e têm como referência as publicações oficiais internacionais constantes na tabela 1. 
Tabela 1. Valores orientadores de material a ser dragado recomendado pela Resolução CONAMA N 454/2012.

\begin{tabular}{|c|c|c|c|c|}
\hline \multirow{4}{*}{$\begin{array}{r}\text { POLUENTES } \\
\text { Metais e } \\
\text { Semimetais }\end{array}$} & \multicolumn{4}{|c|}{ NÍVEIS DE CLASSIFICAÇÃO DO } \\
\hline & \multicolumn{4}{|c|}{ MATERIAI A SER DR AFADO (am unidade de material ceco) } \\
\hline & \multicolumn{2}{|c|}{ ÁGUA DOCE } & \multicolumn{2}{|c|}{ ÁGUA SALINA/SALOBRA } \\
\hline & Nível & Nível & Nível & Nível \\
\hline Arsênio (As) & 5,9 & 17 & 19 & 70 \\
\hline Cádmio (Cd) & 0,6 & 3,5 & 1,2 & 7,2 \\
\hline Chumbo $(\mathrm{Pb})$ & 35 & 91,3 & 46,7 & 218 \\
\hline Cobre $(\mathrm{Cu})$ & 35,7 & 197 & 34 & 270 \\
\hline Cromo $(\mathrm{Cr})$ & 37,3 & 90 & 81 & 370 \\
\hline Mercúrio (Hg) & 0,17 & 0,486 & 0,3 & 1,0 \\
\hline Níquel (Ni) & 18 & 35,9 & 20,9 & 51,6 \\
\hline Zinco (Zn) & 123 & 315 & 150 & 410 \\
\hline
\end{tabular}

Fonte: CONAMA (2012, 14p)

O uso de métodos considerados "pseudototais" se justifica pelo fato de estes possibilitarem a determinação da influência antropogênica e, dessa forma, poderem ser usados no monitoramento ambiental (SCANCAR et al., 2000; ALLOWAY, 2012). Por outro lado, as extrações consideradas “totais" produzem poucas informações úteis, uma vez que os efeitos ecotoxicológicos de um elemento químico, assim como o seu comportamento ambiental (transporte, reatividade, mobilidade etc), dependem totalmente da sua forma química (KOT e NAMIESNIK, 2000).

No Brasil, a Resolução n 420 do CONAMA, de 28 /12/2009, que "dispõe sobre critérios e valores orientadores de qualidade do solo quanto à presença de substâncias químicas e estabelece diretrizes para o gerenciamento ambiental de áreas contaminadas por essas substâncias em decorrência de atividades antrópicas" também recomenda os dois tipos supracitados de metodologias de extração das substâncias inorgânicas das amostras (USEPA 3050 ou USEPA 3051 em suas atualizações).

Os critérios de avaliação da qualidade dos solos e sedimentos se aproximam bastante, isso porque tais protocolos são cada vez mais relacionados às informações biológicas (AHLF et al., 2002). Os solos e sedimentos são compostos essencialmente das mesmas matrizes, eles recebem inputs semelhantes de contaminantes, onde os principais processos interativos entre os componentes sólidos e dissolvidos no geral diferem apenas na intensidade (AHLF e FÖRSTNER, 2001), justificando essas similaridades nos protocolos supracitados. 
Com isso, é valido levar em consideração os métodos utilizados para se estabelecer os valores de referência de qualidade (VRQs) do solo quanto à presença de substâncias químicas quando da elaboração desses VRQs para sedimentos estuarinos.

\section{CONSIDERAÇÕES FINAIS}

Em virtude da crescente pressão antrópica sobre as áreas estuarinas a determinação de valores de background geoquímico para elementos químico (maiores, menores e traços) trazem implicações significativas para a gestão desses ambientes, sobretudo quando se trata de avaliar se há a ocorrência de contaminação no presente, ou para se usar como base de comparação para o futuro.

Cabe ressaltar que há áreas estuarinas onde o substrato geológico é o fator que mais influencia nas concentrações naturais de elementos químicos nos sedimentos. Isso mostra a necessidade de se estabelecer valores orientadores de forma local/regional com o propósito de se evitar sub ou superestimação de grau de contaminação dos ambientes estuarinos.

O conhecimento desses valores, especialmente no que diz respeito aos elementos químicos tóxicos, serve como subsídio aos gestores para o planejamento de suas ações corretivas e/ou mitigadoras, e o monitoramento futuro desses ambientes tão sensíveis à ação humana.

\section{AGRADECIMENTOS}

A primeira autora agradece a CAPES pela bolsa de doutorado.

\section{REFERÊNCIAS}

ABLE, K.W.; FAHAY, M.P. Ecology of Estuarine Fishes: Temperate Waters of the Western North Atlantic. Baltimore, MD: Johns Hopkins University Press. 2010.

AHLF W.; FÖRSTNER U. Managing Contaminated sediments, Part I: Improving chemical and biological criteria. J Soils Sediments. v.1, p.30-36, 2001.

AHLF, W.; BRAUNBECK, T.; HEISE, S.; HOLLERT, H. Sediment and soil quality criteria. In: BURDEN, F.R., MCKEVIE, I., FORSTNER, U., GUNTHER, H. (Eds.). Environmental Monitoring Handbook. New York: McGraw-Hill Professional, 2002.1-17. 
ALEXAKIS, D. Geochemistry of stream sediments as a tool for assessing contamination. European Water. v. 21/22, p. 57-72, 2008.

ALLOWAY, B.J. Heavy metals in soils. 3.ed. Dordrecht: Springer. 2012.

AMORIM, A.; RAMO, S.; MICHAEL, E.; FRANC, A.; BORDALOAB, A. Habitat loss and gain: Influence on habitat attractiveness for estuarine fish communitie. Estuar Coast Shelf Sci. v.15, p. 244-257, 2017

BIANCHI, THOMAS S. Biogeochemistry of estuaries. Oxford: Oxford University Press. 2007. BIRCH, G.F., CHANG, C.H., LEE, J.H., CHURCHILL, L.J. The use of vintage surficial sediment data and sedimentary cores to determine past and future trends in estuarine metal contamination (Sydney Estuary, Australia). Sci Total Environ. v. 1, p. 542-561, 2013.

CALMANO, W., HONG, J.; FÖRSTNER, U. Binding and mobilization of heavy metals in contaminated sediments affected by $\mathrm{pH}$ and redox potential. Water Sci. Technol. v. 28, n. 8-9, p. 223-235, 1993.

CHESTER, R.; JICKELLS, T. D. Marine Geochemistry. 3.ed. Oxford: Wiley-Blackwell. 2012. CHURCH, T.M.; SCUDLARK, J.R. Trace metals in estuaries: A Delaware Bay synthesis. In: ALLEN, H.E., GARRISON, A.W., LUTHER, G.W. (Eds.). Metals in Surface Waters. Chelsea MI: Ann Arbor Press, MI. 1998. p.1-22.

CONAMA - Conselho Nacional do Meio Ambiente. Resolução $\mathbf{N}^{\mathbf{0}}$ 454, de 1 de novembro de 2012. Disponível em:

http://www.mma.gov.br/port/conama/processos/E4B1AB8F/PropResol_VLIMPA_Dragagem_3a CTAJ.pdf >. Acesso em: 04 de outubro de 2012.

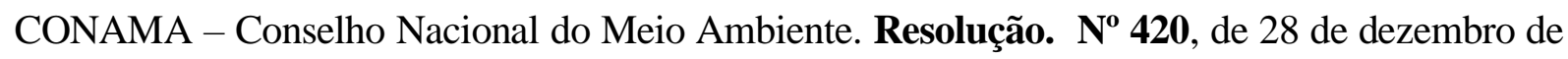
2009. Disponível em: http://www.mma.gov.br/port/conama/legiabre.cfm?codlegi=620. Acesso em: 04 de outubro de 2012.

DAY JR, J. W.; YANEZ-ARANCIBIA, A.; KEMP, W. M.; CRUMP, B. C. Introduction to estuarine ecology. In: DAY JR, J. W.; YANEZ-ARANCIBIA, A.; KEMP, W. M.; CRUMP, B. C. Estuarine ecology. New Jersey: Wiley-Blackwell. 2013. p. 1-18.

DEPAULA, F. C.; MOZETO, A. A. Biogeochemical evolution of trace elements in a pristine watershed in the Brazilian southeastern coastal region. Appl Geochem. Oxford, v. 16, n. 9, p. 1139-1151, 2001. 
FÖRSTNER, U. Traceability of sediment analysis. Trends Analyt Chem.v. 3, p. 217-236, 2004.

GAŁUSZKA A, MIGASZEWSKI Z.M. Geochemical background-an environmental perspective. Mineralogia. v. 42, n. 1, p. 7-17, 2011.

GALUSZKA, A. Different approaches in using and understanding the term "Geochemical Background"- pratical implications for environmental studies. Pol J Environ Stud. v. 16, n. 3, p. 389-395, 2007.

GAŁUSZKA, A. Methods of determining geochemical background in environmental studies. Prob.Ekol.Kraj. v. 16, n. 1, p. 507-519, 2006.

HONG S., CANDELONE J.P., PATTERSON C.C., BOUTRON C.F. Greeland ice evidence of hemispheric lead pollution two millennia ago by Greek and Roman civilizations. Science. v. 265, n. 5180, p. 1841-1843, 1994.

HONG, S.; CANDELONE, J.; P. PATTERSON, C. C.; BOUTRON, C. F. History of Ancient copper smelting pollution during Roman and Medieval times recorded in Greenland ice sheet. Science. V. 272, p. 246-248, 1996.

JENNE, E.A. Controls on $\mathrm{Mn}, \mathrm{Fe}, \mathrm{Co}, \mathrm{Ni}, \mathrm{Cu}$ and $\mathrm{Zn}$ concentrations in soils and water: the significant role of hydrous Mn and Fe oxides. Amer. Chem. Soc. v. 73, p. 337-387, 1968.

JESUS, H. C.; COSTA, E. A.; ZANDONADE, E.; GONÇALVES, S. M. Distribuição de metais pesados em sedimentos do sistema estuarino da ilha de Vitória-ES. Quím. Nova. v. 27, n. 3, p.378386, 2004.

KENNISH, M. J. Pollution in estuaries and coastal marine waters. J. Coastal Res. v. 12, p. 27-49, 1994.

KOT, A. AND NAMIESNIK, J. The Role of Speciation in Analytical Chemistry. Trends Analyt Chem. v. 19, p. 69-79, 2000.

LACERDA A.D., MARINS R.V. Antrhopogenic mercury emissions to the atmosphere in Brazil: The impact of gold mining. J. Geochem. Exploration. v. 58, p. 223-229, 1997.

LI, B., FENG, C., LI, X., CHEN, Y., NIU, J., SHEN, Z. Spatial distribution and source apportionment of PAHs in surficial sediments of the Yangtze Estuary, China. Mar Pollut Bull. v. 64, p. 636-643, 2012. 
LIMA FILHO, M. F. Análise Estratigráfica e Estrutural da Bacia Pernambuco. Tese de doutorado em Geoquímica e Geotectônica. IGC/USP, São Paulo, 1998.

LOURIÑO-CABALA, B., LEVSEN, L., CHARRIAU, A., BILlON, G., OUDDANE, B., \& BOUGHRIET, A. Potential risks of metal toxicity in contaminated sediments of Deûle river in Northern France. J Hazard Mater. v. 186, p. 2129-2137, 2011.

LUIZ-SILVA, W., MATOS, R. H. R., KRISTOSCH, G. C., \& MACHADO, W. Variabilidade espacial e sazonal da concentração de elementos-traço em sedimentos do sistema estuarino de Santos-Cubatão (SP). Quím. Nova. v. 29, n. 2, p. 256-263, 2006.

MATSCHULLAT, J., OTTENSTEIN, R., \& REIMANN, C. Geochemical background-can we calculate it? Environ Geol. v. 39, n. 9, p.990-1000, 2000.

MENDES, N. M. T. N. S. Background geoquímico para sedimentos de regiões estuarinas de Pernambuco. 2015. Tese de doutorado em Geociências. CTG/UFPE, Recife, 2015.

MIRANDA, L. B.; CASTRO, B. M.; KJERFVE, B. Princípios de oceanografia Física de estuários. São Paulo: Edusp, 2002.

MIRLEAN, N. BAISCH, P., TRAVASSOS, M.P., NASSAR, C. Calcareous algae bioclast contribution to sediment enrichment by arsenic on the Brazilian subtropical coast. Geo- mar. Lett. v. 31, n, 1, p. 65-73, 2011.

NASCIMENTO, M.A.L. Geologia, geocronologia, geoquímica e petrogênese das rochas ígneas cretácicas da província magmática do Cabo e suas relações com as unidades sedimentares da Bacia de Pernambuco, NE do Brasil. Tese de doutorado em Geodinâmica e Geofísica. CCET/ UFRN, Natal, 2003.

OLIVEIRA, R. C. B; MARINS, R. V. Dinâmica de Metais-Traço em Solo e Ambiente Sedimentar Estuarino como um Fator Determinante no Aporte desses Contaminantes para o Ambiente. Rev. Virtual Quim. v. 3, n. 2, p. 88-102, 2011.

PASLAWSKI, P. \& MIGASZEWSKI, M. The quality of element determinations in plant materials by instrumental methods. Polish J. Environ. Stud. v. 5, n. 2a, p. 154-164, 2006.

PERIN, G.; FABRIS, R.; MANENTE, S.; WAGENER, R, A.: HAMACHER, C.; SCOTT, S. A Five-Year Study on the Heavy Metal Pollution of Guanabara Bay Sediments (Rio de Janeiro, Brazil) and Evalution of the Metal Biovailability by Means of Geochemical Speciation. Wat. Res. v. 31, n. 12, p. 3017-3028, 1997. 
POTTER, I.C. TWEEDLEY, J.R.; ELLIOTT, M.; WHITFIELD, A.K. The ways in which fish use estuaries: a refinement and expansion of the guild approach. Fish Fish., v. 16, p. 230-239, 2015.

REIMANN C.; CARITAT P. Chemical elements in the environment - factsheets for the geochemist and environmental scientist. Germany: Springer Verlag, 1998.

REIMANN, C., GARRET, R.G. Geochemical background - concept and reality. Sci. Total Enviro. v. 350, p. 12-27, 2005.

REIMANN, C., FILZMOSER, P., GARRETT, R.G. Background and threshold: critical comparison of methods of determination. Sci. Total Enviro. v. 346, p. 1-16, 2005.

ROMERO, F.M., ARMIENTA, M.A. \& CARRILLO, C. A. Arsenic sorption by carbonate- rich aquifer material, a controlo n arsenic mobility at Zimapan. Mexico. Arch. Environ. Contam. Toxicol. v. 47, p. 1-13, 2004.

SABADINI-SANTOS, E., KNOPPERS, B. A., OLIVEIRA, E. P., LEIPE, T., \& SANTELLI, R. E. Regional geochemical baselines for sedimentary metals of the tropical São Francisco estuary, NE-Brazil. Mar Pollut Bull. v. 58, n. 4, p. 601-606, 2009.

SANDERS, L. M., SANDERS, C. J., LUIZ-SILVA, W., MACHADO, W., SILVA-FILHO, E. V., PATCHINEELAM, S. R. Anthropogenic source assessment of $226 \mathrm{Ra}$ and $210 \mathrm{~Pb}$ in a sediment core from the Cubatão River estuary (SE Brazil). J Radioanal Nucl Chem. v. 287, p. 729-732, 2011.

SCANCAR, J.; MILACIC, R.; STRAZAR, M.; BURICAO. Total metal concentrations and partioning of Cd, Cr, Cu, Fe, Ni and Zn in sewage sludge. Sci Total Environ. v. 250, p. 9-19, 2000. TAN, K. H. Humic Matter in Soil and the Environment: Principles and Controversies. 2. Ed. New York: CRC Press, 2014.

TURNER, A., S. M.; G. E. MILLWARD. Suspended particles: Their role in estuarine biogeochemical cycles. Estuar. Coast. Shelf Sci. v. 55, p. 857-883, 2002.

U.S. EPA. Method 3050B: Acid Digestion of Sediments, Sludges, and Soils Revision 2. Washington, DC, 1996.

U.S. EPA. Method 3051A (SW-846): Microwave Assisted Acid Digestion of Sediments, Sludges, and Oils. Revision 1. Washington, DC, 2007. 
U.S. EPA. Method 7471B (SW-846): Mercury in Solid or Semisolid Wastes (Manual Cold-Vapor Technique). Washington, DC, 2007.

VAIPHASA, C., SKIDMORE, A.K. E DE BOER, W.F. A post-classifier for mangrove mapping using ecological data. ISPRS J Photogramm Remote Sens. v. 61, p. 1-10, 2006. 